\title{
Alexander von Humboldt und Frankreich
}

Von Heinz Balmer

Alexander von Humboldts Beziehungen zu Frankreich beginnen mit seiner Mutter. Sie war eine geborene Colomb aus der französischen Kolonie bei Berlin. Die französische Literatur galt als führend. Als Humboldt 18jährig war, erschien Bernardin de Saint-Pierres «Paul et Virginie». Dessen Naturschilderungen hat er geschätzt. Er berichtet davon im zweiten Bande des «Kosmos» ${ }^{1}$. Mit 19 Jahren sah er 1788 in Berlin den Ballonaufstieg des Franzosen Jean-Pierre Blanchard ${ }^{2}$. Zum erstenmal aber überschritt er die französische Grenze 1790, als er Georg Forster auf der Reise an den Niederrhein begleitete. Die beiden besuchten anschließend England, und auf der Rückreise fuhren sie von Dover nach Calais und zogen weiter nach Paris. Dort verbrachten sie die erste Juliwoche und erlebten das Nachspiel der Französischen Revolution, die Begeisterung der Massen, die Gesänge des Volkes. Humboldt meinte später, diese kurze Spanne seines Lebens werde ihm «immer die lehrreichste und unvergeßlichste sein ${ }^{3}$ Zum Gebäude der Nationalversammlung, des Tempels der Freiheit, hat er noch selber mit dem Schubkarren Sand geführt.

Als junger Oberbergrat in Franken unternahm er mit seinem Freund Johann Karl Freiesleben 1795 eine Reise in die französischen Alpen. Sie gelangten über Bern und Genf in das Tal von Chamonix und kehrten über den Großen St. Bernhard und Vevey und Bern wieder zurück.

1796 hatten die Franzosen Württemberg erobert. Der preußische Minister Hardenberg bat Humboldt, er möge dort beim kommandierenden General Moreau vorsprechen und sich zusichern lassen, daß die Franzosen nicht etwa auch das angrenzende preußische Fürstentum Ansbach beträten. Humboldt erfüllte diesen Auftrag ${ }^{4}$.

Nach dem Tode seiner Mutter erbte er ein großes Vermögen, so daß er 1797 seine Stelle als Bergingenieur niederlegte und sich 1798 nach Paris begab, wo sein Bruder Wilhelm und dessen Frau sich schon seit dem Winter aufhielten. Alexander wollte sich auf eine Forschungsreise vorbereiten. Er wußte nicht, wie man einen Sextanten bedient, um Sonnen- und Sternhöhen abzulesen, und wandte sich daher an die Astronomen der Pariser Sternwarte. Lalande und Delambre nahmen den preußischen Adligen freundlich auf. Sie berieten ihn beim Ankauf der Instrumente und unterwiesen ihn in ihrer Handhabung ${ }^{5}$. Borda forderte ihn auf, eine magnetische Neigungsnadel mitzunehmen und ihre Schwingungen in zehn Minuten zu zählen. An Orten, wo die erdmagnetische Intensität größer ist, 


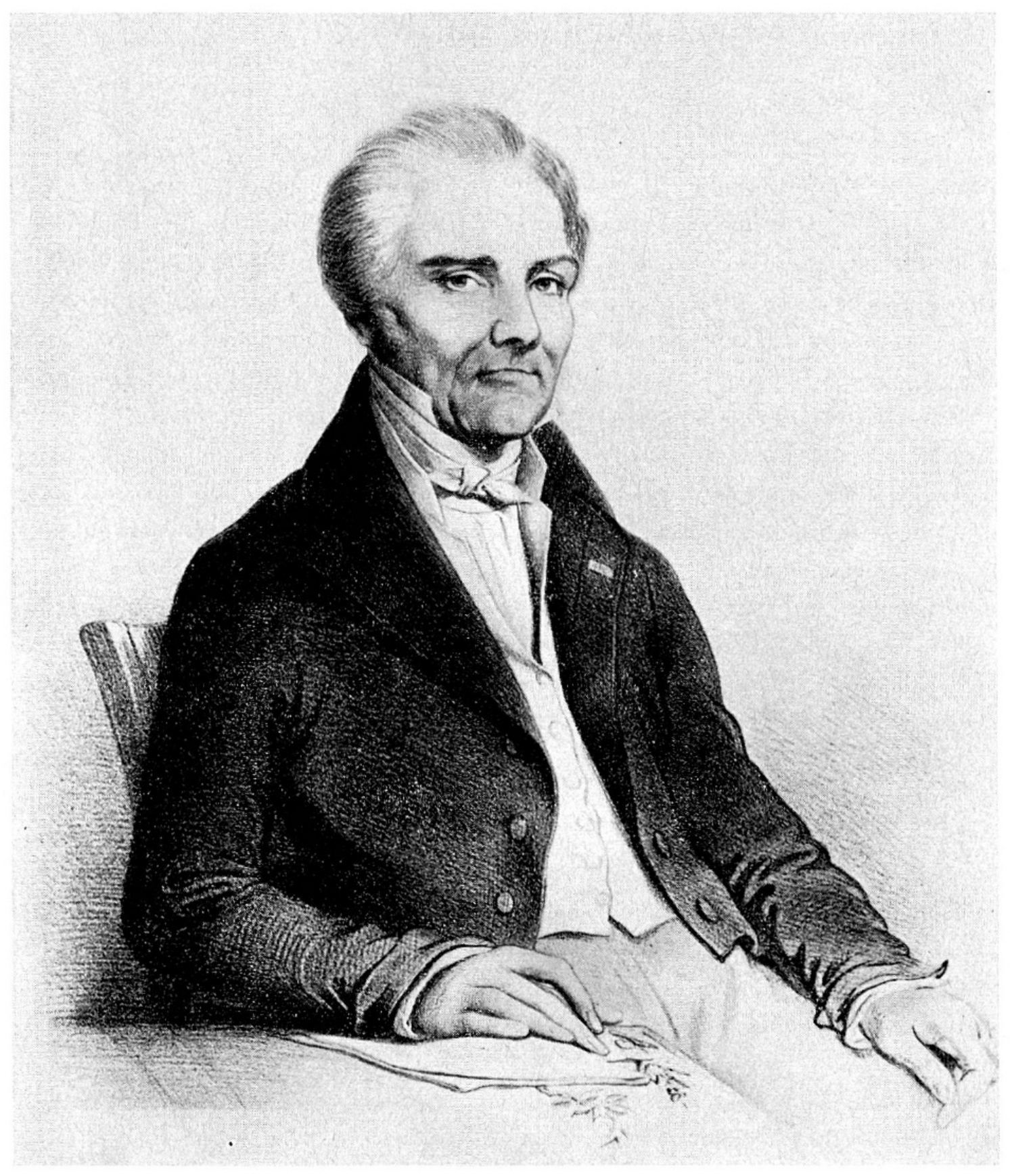

Aimé Bonpland (1773-1858) 
schwingt diese Nadel schneller, und wo die einwirkende Kraft schwächer ist, oszilliert sie langsamer.

Humboldt war damals vor allem als Chemiker tätig. Über seine Ergebnisse hielt er mehrere Vorträge in der Académie des Sciences des «Institut National», so über das Verhältnis von Stickstoff und Sauerstoff in der atmosphärischen Luft. Er lernte die führenden Chemiker kennen, Fourcroy, Vauquelin, Thenard, Chaptal. Anfangs Juni 1798 erlebte er die Vollendung der französischen Gradmessung mit $^{6}$. Französische Forscher hatten von Dünkirchen bis Barcelona einen Meridianbogen gemessen, um daraus die Erdgröße zu bestimmen.

Humboldt wohnte in Paris im Hotel Boston an der Rue Colombier 7. Wenn er am Abend heimkehrte, begegnete ihm oft ein Pflanzensammler, der mit einer zerbeulten Botanisiertrommel von seinen Streifzügen kam ${ }^{7}$. Der junge Mediziner erwies sich als Schüler des botanischen Reisenden Louis-Claude-Marie Richard und hieß Aimé Bonpland. Reiselust und republikanische Gesinnung führten Humboldt und Bonpland zusammen. Am 20.Oktober 1798 verließen sie Paris und erreichten in einer Woche Marseille, um nach Nordafrika zu segeln. Aber es nahm sie in dieser Kriegszeit kein Schiff mit. Auf eine Gelegenheit wartend, sammelten sie Pflanzen, Krebse und Muscheln oder übten sich in magnetischen, meteorologischen und astronomischen Beobachtungen. Mitte Dezember entschlossen sie sich, nach Spanien zu ziehen, und von dort konnten sie 1799 zur Forschungsreise in die Tropengegenden Amerikas aufbrechen.

Nach über fünf Jahren trafen sie am 1. August 1804 in der Garonnemündung bei Bordeaux wieder ein. Während Bonpland zu seinem Bruder nach La Rochelle eilte, verstaute Humboldt sein Gepäck in der Diligence und reiste nach Paris. Schon sechs Wochen später erstattete er im Institut einen ersten Bericht; er zeigte Karten, Zeichnungen neuer Pflanzen, exotischer Tiere und Landschaften. Im Jardin des Plantes stellte er im Oktober Objekte aus, die in 35 Kisten inzwischen eingetroffen waren. Humboldt entfaltete seine Vielseitigkeit als Botaniker, Zoologe, Chemiker, Physiker, Meteorologe, Astronom und Geograph, so daß der große Chemiker Berthollet ausrief: "Cet homme réunit toute une Académie en lui.» ${ }^{8} \mathrm{Im}$ September entstand die Zeichnung, die der Maler François Gérard von Humboldt anfertigte, und im Oktober wurde er Napoleon vorgestellt. Dieser bereitete sich zur Kaiserkrönung vor und liebte den preußischen Baron nicht, der einen Teil der öffentlichen Aufmerksamkeit auf sich ablenkte. Er hielt ihn auch später für einen Spion.

Humboldt und Bonpland hatten schon von Amerika her Samen nach Frankreich geschickt. Duftende Jasmine in den Gärten, schmückende Kamelien in den Salons waren Mode geworden ${ }^{9}$. Bonpland wurde nach Schloß Malmaison ab- 
geordnet, um der Kaiserin als Park- und Gartengestalter zu dienen. Die Schreibtischarbeit zur Auswertung seiner reichen amerikanischen Sammlung lag ihm weniger. Humboldt ließ 1813 in der Not den Neffen seines einstigen Hofmeisters kommen, den Berliner Botaniker Carl Sigismund Kunth, der mit ihm die 14 Bände der Pflanzenbeschreibung ausarbeitete. Bonpland kehrte 1816 nach Südamerika zurück. Er wollte den Paraguay erforschen, wurde jedoch gefangengesetzt und trotz den Bitten Humboldts erst 1829 freigelassen. Er lebte dann in Uruguay als Arzt bei Indianern und starb dort als armer Greis ein Jahr vor Humboldt ${ }^{10}$.

Während Napoleon das Blut des Landes vergoß, blühten in der Hauptstadt die Künste des Friedens. Paris war damals der Mittelpunkt der Wissenschaft. Dort fand Humboldt, wessen er zur Auswertung der Reise bedurfte: Gelehrte, die den Stoff bearbeiten halfen, Kupferstecher, Verleger ${ }^{11}$.

Wenn man zusammenrechnet, wie lange Humboldt im ganzen in Paris weilte, erhält man eine Summe von 22 Jahren und vier Monaten. Davon entfällt der Hauptanteil auf die Zeit zwischen 1807 und 1826, während die ganze Spanne vom Juli 1790 bis zum Januar 1848 reicht $^{12}$. Man weiß auch, wo er der Reihe nach gewohnt hat: 1804-1805 an der Rue des Augustins; einige Zeit teilte er ein Zimmer mit Gay-Lussac an der Ecole Polytechnique; dann wohnte er an der Rue de la Grange Battelière, 1808 an der Rue St-Dominique d'Enfer 20, 1809 an der Rue de la vieille Estrapade 11; 1811 zog er an die Rue d'Enfer 67, 1813 an den Quai Malaquais 3, 1818 an den Quai de l'Ecole 26, wo er bis 1827 blieb. Mit Hilfe der Aufeinanderfolge dieser Adressen lassen sich zuweilen Briefautographen Humboldts datieren, auf denen die Jahrzahl fehlt, und das ist nicht selten. Er schrieb «Ce Mardi» oder ähnlich.

Das Reisewerk, das in Paris entstand, heißt «Voyage aux régions équinoctiales du Nouveau Continent». ${ }^{13}$ Die dreißig Bände erschienen von 1805 bis 1834. Sechs Teile lassen sich unterscheiden, erstens die Beschreibung der Reise und die Geschichte früherer Entdeckungsfahrten (7 Bände), zweitens Zoologie (2 Bände), woran der Fischforscher Valenciennes und der Insektenforscher Latreille und auch Cuvier mitwirkten, drittens Geographie und Politik Mexikos (3 Bände), viertens die astronomischen, barometrischen, trigonometrischen und physikalischen Ergebnisse (3 Bände), fünftens Pflanzengeographie (ein Einzelband, der 1805 als erster das ganze Werk eröffnete und von grundlegender Bedeutung war), sechstens Botanik (14 Folianten mit zusammen 1240 Kupfertafeln). Humboldt hatte gehofft, in drei Jahren fertig zu sein; daraus wurden fast dreißig. Indem er nicht nur die Honorare für Kupferstecher und Mitarbeiter, sondern auch den Druck bezahlte, opferte er sein ganzes Vermögen. 
Die Vielfalt seines Werkes brachte ihn zu den verschiedensten Gelehrten in Beziehung. U̇ber die Mathematiker hat er später einmal dem Berliner Mathematiker Jacobi geschrieben: «Bei einer sehr geringen mathematischen Kenntnis, bei der Unwissenheit, die ich so gern eingestehe, hat mir doch der lange Umgang mit Lagrange, Laplace und Fourier einiges Ahndungsvermögen über den relativen Wert meiner Zeitgenossen eingeflößt. ${ }^{14}$ Durch Lagrange wurde er auf den jungen Gauß in Göttingen aufmerksam, und dieser ist für den alten Humboldt die für Mathematik zuständige Stelle geblieben. Sooft er ein mathematisches Genie entdeckt zu haben glaubte, klopfte er dort an. Gauß ließ sich eigentlich nicht gerne stören, war aber immer hilfsbereit, und hie und da konnte er Humboldts Vermutung bestätigen. Felix Klein hat Humboldt deswegen in seinen «Vorlesungen über die Entwicklung der Mathematik im 19. Jahrhundert» einen eigenen Abschnitt gewidmet. Humboldt habe die seltene Gabe besessen, auch ohne genaues Verständnis die Bedeutung ihm ferner liegender Gebiete zu erkennen, schreibt Klein ${ }^{15}$. - Zu den Biologen hatte er ebenfalls Beziehungen: zu den Pflanzenforschern Antoine-Laurent de Jussieu und René Louiche Desfontaines, zu den Zoologen Lamarck, Geoffroy Saint-Hilaire und ebenso zu deren Gegner Cuvier, dessen Einfluß er benutzte, um aufstrebende Talente zu fördern. Auch die Gelehrten, die mit Napoleon 1798 Ägypten besucht hatten oder sich an der Auswertung jener Reise beteiligten, standen ihm nahe, vor allem der Geograph und Archäologe Edme-François Jomard und die Brüder Champollion. Als der Entzifferer der Hieroglyphen 1832 starb, trug Humboldt einen Zipfel des Bahrtuches. Beim Altertumsforscher Letronne hörte er noch 1831 Vorlesungen ${ }^{16}$.

Eines der Ergebnisse von Humboldts Reise war die Beobachtung, daß Bordas Neigungsnadel am magnetischen Äquator in Peru am langsamsten geschwungen hatte und daß sie, wenn man sich davon entfernte, von Zone zu Zone schneller um die Ruhelage schwankte. Der Physiker Jean-Baptiste Biot bemühte sich, die Ergebnisse mathematisch zu fassen. Im Dezember 1804 konnten sie ihre gemeinsame Abhandlung dem Institut vorlegen.

Besonders verbunden war Humboldt unter den Chemikern mit Berthollet, Gay-Lussac und Thenard ${ }^{17}$. Er nannte sie Ammoniak, Pottasche und Soda, weil sie darüber gearbeitet hatten. Berthollet war eine Generation älter als die beiden andern. Er besaß ein Landhaus in Arcueil und empfing dort Gelehrte; er nannte den Kreis «Société d'Arcueil» und gab eine Abhandlungsreihe, die «Mémoires d'Arcueil», heraus. Diesem Zeitgenossen zu Ehren hat Humboldt ein botanisches Geschlecht «Bertholletia» genannt; sein größter Vertreter ist die Bertholletia excelsa, der mächtigste Baum des brasilianischen Urwaldes. Eines Tages sah Humboldt in Arcueil einen jungen Mann von hohem Wuchs. «Es ist 
Gay-Lussac», sagte man ihm, «jener, der neulich im Ballon zur größten bisher erreichten Höhe der Atmosphäre aufgestiegen ist.» - «Es ist», fügte Humboldt selbst hinzu, «der Verfasser der herben Kritik meiner Arbeit über die Messung des Luftsauerstoffs. » Dann aber näherte er sich ihm, grüßte ihn herzlich und bot ihm seine Freundschaft an ${ }^{18}$. Bald beschlossen sie, den Sauerstoffgehalt der Luft gemeinsam zu bestimmen. - Phosphor, Salpetergas, Wasserstoffgas haben die Eigenschaft, Sauerstoff aufzunehmen. Wenn man eine gemessene Luftmenge in einem Glasrohr damit einschließt, etwa Luft mit Phosphor über Quecksilber absperrt, so wird der Sauerstoff verzehrt, und aus der Volumenverminderung läßt sich der Sauerstoffanteil ablesen. Humboldt und Gay-Lussac stellten fest, daß Voltas Verfahren das genaueste sei. Dieser ließ durch ein Gemisch aus Luft und überschüssigem Wasserstoffgas Funken springen; dabei verbindet sich der Sauerstoff der Luft mit dem Wasserstoff zu Wasser. Die Freunde entdeckten 1805, daß es einfacher ist, statt auf die Gewichtsteile auf die Volumina zu achten, weil sich mit dem einfachen Volumen Sauerstoffgas gerade ein doppeltes Volumen Wasserstoffgas verbinde ${ }^{19}$. Da zwei Drittel des verbrannten Gasvolumens aus Wasserstoff und ein Drittel aus Sauerstoff besteht, ergibt sich die gesuchte Sauerstoffmenge, indem man die eingetretene Volumenverminderung durch drei dividiert. $\mathrm{Daß}$ sich allgemein Gase in einfachen Volumenverhältnissen verbinden, wies GayLussac 1808 in einer zweiten Arbeit nach.

Mit Gay-Lussac zusammen hat Humboldt 1805 auch seine erste Italienreise unternommen. Auf den Alpen oben sammelten sie Luft in Flaschen. In Rom besuchten sie Alexanders Bruder Wilhelm, der dort preußischer Gesandter war, und in Neapel erlebten sie zusammen mit dem Geologen Leopold von Buch einen Ausbruch des Vesuvs.

Die Gradmessung wurde von Barcelona noch bis zu den Balearischen Inseln erweitert. Biot und Arago wurden 1806 vom Längenbureau damit beauftragt. Als 1808 die Franzosen in Spanien einrückten, weilte Arago auf dem Berg über dem Hafen der Insel Mallorca ${ }^{20}$. Unter dem Gerücht, er gebe den Truppen Landungssignale, wurde er gefangengesetzt, entkam aber auf einem Schiff in die nordafrikanische Küstenstadt Algier. Mit zwei Löwen, die der Dey von Algier an Napoleon schickte, fuhr Arago am 13. August 1808 gegen Marseille. Eine spanische Raubschaluppe fing das Schiff und verschleppte es nach Rosas auf der spanischen Seite der Pyrenäen. Drohungen des Deys erwirkten die Freigabe; aber ein Sturm verschlug das Schiff südwärts bis an die Küste Afrikas. Arago erreichte abermals Algier und nach erneuter Überfahrt endlich am 2. Juli 1809 das Quarantänegebäude von Marseille. Dort empfing er als ersten Brief den Glückwunsch Alexander von Humboldts, der Arago nicht kannte, aber von Lagrange sein Lob 


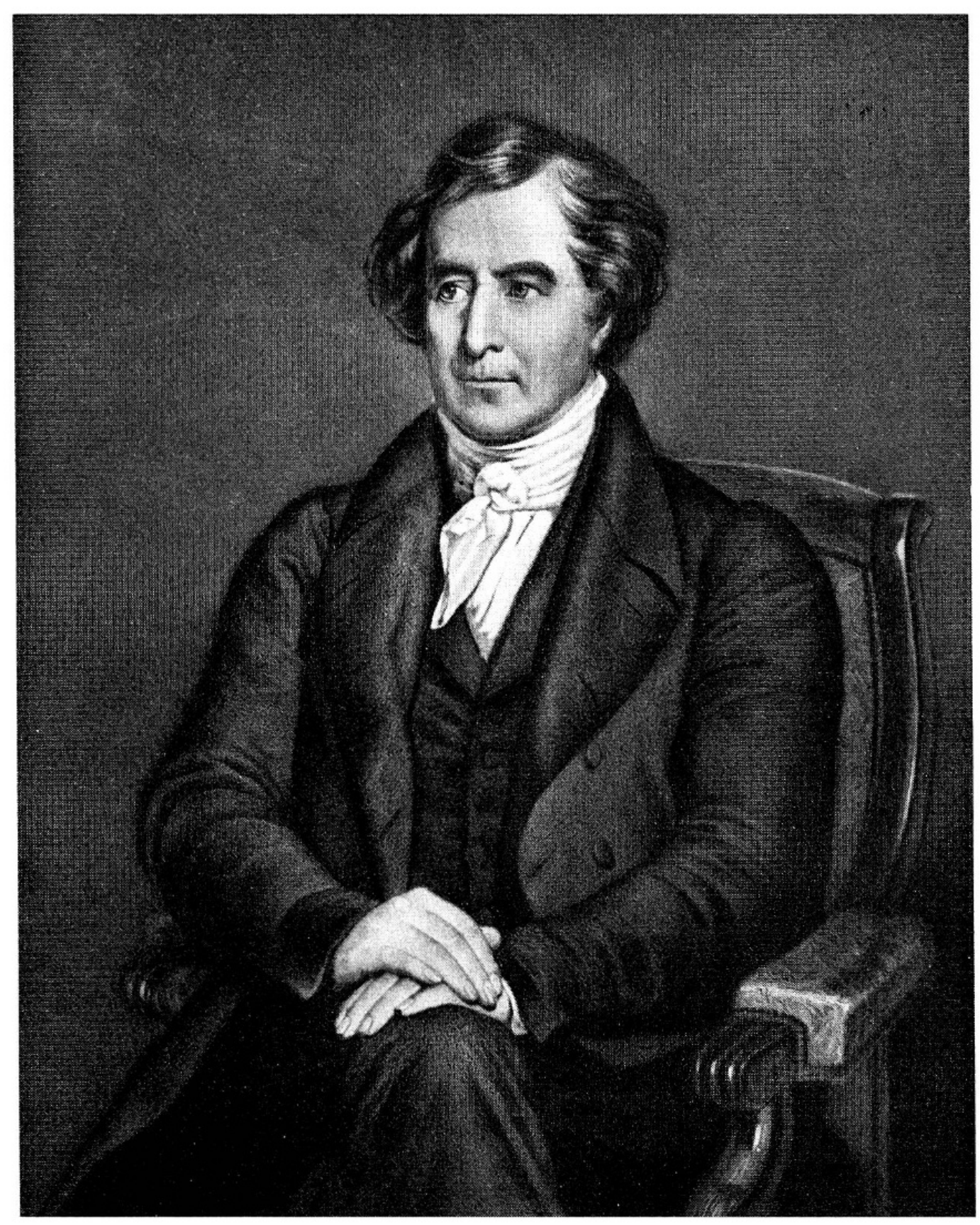

François Arago (1786-1853) 
vernommen und die Abenteuer mit Teilnahme verfolgt hatte. Dies war der Anfang einer Freundschaft, die bis zu Aragos Tode 1853 dauerte. Seit 1830 war Arago Direktor der Pariser Sternwarte und hielt dort immer ein Zimmer für Humboldt bereit. Gelegentlich gerieten sie in heftigen Wortwechsel; aber das war bei ihrem Feuer ganz natürlich. Politisch waren beide Demokraten, Arago noch entschiedener als Humboldt; in der Reaktionszeit kämpfte er für Gedankenfreiheit. Humboldt bewunderte seinen Stil, seinen Arbeitseifer und seinen Mut ${ }^{21}$. Einmal schrieb er über Arago, er sei «eine der edelsten Menschennaturen, in der Weisheit und Güte gepaart sind $»,{ }^{22}$ und ein andermal nannte er ihn «la personne qui m'est la plus chère dans cette vie $»{ }^{23}$ Arago nahm kein Blatt vor den Mund und beleidigte manchmal Leute; dann nahm ihn Humboldt in Schutz: «Monsieur Arago est vif, mais bon et chaud de caractère. ${ }^{24}$ Als einst der Kabinettsrat Markus Niebuhr am preußischen Hof über Arago schimpfte, flocht Humboldt ein: «Und doch ist dieses Ungeheuer der liebste Freund, den ich in Frankreich habe. » ${ }^{25}$ Außer mit Jugendfreunden duzte er sich nur mit Bonpland, Gay-Lussac, Arago und dem Mathematiker Poisson ${ }^{26}$. 1839 erzählt Varnhagen in seinem Tagebuch, Humboldt habe ihm ein schönes Arago-Bildnis (von Henry Scheffer) gezeigt, und von 1856 wissen wir, daß eine Büste Aragos in einer Fensternische seines Studierzimmers stand ${ }^{27}$. Unmittelbar nach Aragos Tode wurden seine Werke in 16 Bänden französisch und deutsch herausgegeben. Der 84jährige Humboldt schrieb die umfangreiche Einleitung in vier Nächten.

Es gibt Berichte über Humboldts Lebensweise in Paris. In den politisch bewegten Jahren 1813 und 1814 fand er Zuflucht im Zeichnen und Malen. Die Porträtisten François Gérard und Karl von Steuben unterrichteten ihn. Ferner lernte er Persisch bei Silvestre de Sacy, denn er plante eine Orientreise über Persien nach Indien.

Er stand früh auf, nahm ein leichtes Frühstück mit Kaffee und Weißbrötchen, ging dann zu Arago und in die Bibliothek des Institut National, wo er bis sieben Uhr abends arbeitete. Ein einfaches Mittagessen in einer benachbarten Gaststätte unterbrach ihn nur kurz. Nachmittags besprach er sich nicht selten mit seinen Druckern und Verlegern. Dann aß er zu Nacht und eilte nach Hause, um sich umzukleiden, denn nun besuchte er Gesellschaften, oft mehrere, bis Mitternacht, und ging dann heim, um noch zwei bis drei Stunden zu arbeiten. Er brauchte nur vier Stunden Schlaf ${ }^{28}$. Stille Forschung und lebhafte Geselligkeit verbanden sich im regelmäßigen Tagesablauf. Roderick Impey Murchison, der englische Geologe, hat gesagt, Humboldt habe in seinem langen Leben mehr geplaudert als irgendeiner seiner Zeitgenossen ${ }^{29}$. Er konnte sechs Stunden en suite sprechen. Wilhelm, der Bruder, schrieb einmal aus London an seine Frau: 
«Es ermüdet auch furchtbar die Ohren, da sein Redefluß unerbittlich dahinrauscht. ${ }^{30}$ Aber ebenso ausdauernd kritzelte seine Feder. Adelbert von Chamisso, der ihn in Paris kennenlernte, schrieb: «Humboldt arbeitet unermüdlich emsig an der Herausgabe seiner Werke... Solche Tätigkeit, Schnelligkeit und Festigkeit ist noch nie gesehen worden. ${ }^{31}$ Mit unerhörtem Einsatz widmete er seine Fürsorge jüngeren Forschern. Als Boussingault eine Südamerikareise plante, unterwies nun er ihn im Gebrauch der Instrumente, schrieb ihm eine «Instruction » und schenkte ihm zwei Barometer ${ }^{32}$.

In der Académie des Sciences hielt er zahlreiche Vorträge ${ }^{33}$. 1804 war er korrespondierendes Mitglied, 1810 eines der acht auswärtigen Mitglieder geworden. Nach dem Reisebericht in vier Fortsetzungen sprach er über Geologie der Kordilleren, über die Schneegrenze, über Pflanzengeographie, über neue Fische, über die atmosphärische Brechung, über die Atmung der Krokodile, über den mexikanischen Kalender, dann über die Affen am Orinoko, die Pflanzenwelt der Kanarischen Inseln, eine neue Vogelart, die Höhlen in Beziehung zum umgebenden Fels, über die Isothermen, die Geographie des Orinoko, galvanische Experimente, die stündlichen Barometerschwankungen, nach 1830 über die Sibirienreise, die Cholera, die indischen Zahlzeichen, das Klima Asiens und über viele andere Gegenstände. Zu den Sitzungen des Instituts nahm er gelegentlich einen Fremdling mit, dem er vom Platz aus munter die Anwesenden erläuterte. Der Berliner Archäologe Gustav Parthey hat aufgeschrieben, was Humboldt ihm am 19. Februar 1821 ins Ohr flüsterte: «Jener kleine Mann mit den äußerst lebhaften Bewegungen ist unser erster Sekretär, Herr Delambre, der die Gradmessung von Dünkirchen bis Barcelona vollendete. Er kann es seinem Vorgänger Lalande noch nicht verzeihen, daß dieser seine Katze als Felis Lalandii unter die Sterne versetzte. - Unser zweiter Sekretär, Herr Cuvier, nach Blumenbach der Begründer der vergleichenden Anatomie, hat ein außerordentliches Verdienst um die französischen Unterrichtsanstalten. Leider ist er ein Mann, den der Ehrgeiz verzehrt, er will durchaus Minister werden. - Der mich soeben begrüßte, ist Herr Poisson, einer unserer ersten Mathematiker und Naturforscher, der sich eben mit seinem eigenen Namen, mit den Fischen, beschäftigt. - Dort in der Ecke sehen Sie meinen intimen Freund Gay-Lussac, dem die Physik ebensoviel verdankt wie die Chemie. Nur durch einen Zufall wurde ich im Jahre 1804 abgehalten, mit ihm zusammen jenen Luftballon zu besteigen, der die bis jetzt größte Höhe über der Erde erreichte. - Jenes ehrwürdige, klassisch gebildete weiße Haupt gehört dem großen Laplace, der in seiner 'Mécanique céleste' noch über Newton hinausging, insofern dies möglich ist. - Bemerken Sie jenen Mann mit dem geistreichen Blick? Es ist der Begleiter des Ersten Konsuls nach Ägypten, Herr 
Fourier, eben jetzt mit tiefsinnigen Untersuchungen über die Wärme beschäftigt. $\aleph^{34}$

Auch bei den Wahlen wurde Humboldt sehr tätig. Achille Valenciennes, der ihm bei den Fischen geholfen hatte und Jahr für Jahr einen eigenen Band über Fische gebar, Valenciennes, der außer für drei eigene Kinder für seine armen Eltern und Schwestern sorgte, ohne daß jemand sich seiner annahm, wurde Humboldts Schützling ${ }^{35}$. Durch ihn erhielt er eine Professur für Anatomie, und 1844 wollte Humboldt ihm den freigewordenen Sitz in der Académie des Sciences verschaffen. Damals war die Académie in zwei Parteien gespalten. Die einen, Anhänger des Königs Louis Philippe, standen unter Führung des Geologen Alexandre Brongniart, die andern, Gegner des Königtums, hörten auf Arago. Humboldt war sowohl mit Brongniart wie mit Arago befreundet. Wenn man also gewählt werden wollte, mußte man sich vor allem bei Humboldt empfehlen. Als Gegenkandidat von Valenciennes wurde der Zoologe Dujardin aufgestellt. Die Partei Brongniarts war für Valenciennes, die Partei Aragos für Dujardin, und man soll sich damals beim Grüßen statt «Wie geht es ?» gefragt haben: «Haben Sie auch einen Brief Humboldts erhalten?» Zu Aragos Anhängern soll Humboldt gesagt haben, was denn schon daran liege, ob ein Esel mehr in der Sektion für Zoologie sitze - kurz, Valenciennes wurde gewählt ${ }^{36}$.

Von Frankreich aus unternahm Humboldt 1805 und 1822 Italienreisen, 1817 und 1818 Englandreisen. Die erste dieser vier Reisen endete in Berlin, das Humboldt seit seiner Amerikareise zum erstenmal wiedersah. Er fand, es sei eine menschenleere Wüste ${ }^{37}$. Der König ernannte ihn zu seinem Kammerherrn; aber Humboldt machte vorläufig keinen Gebrauch davon. «Ich lebe fremd und isoliert in diesem mir fremd gewordenen Lande. ${ }^{38}$ Preußen wurde wenige Monate später unterjocht und von Napoleon bei Jena vernichtend geschlagen. Humboldt, der darunter litt, hat 1808 sein Lieblingswerk, die beiden Bändchen «Ansichten der Natur», deutsch veröffentlicht. «Bedrängten Gemütern sind diese Blätter vorzugsweise gewidmet», schrieb er in der Vorrede ${ }^{39}$.

Die Londoner Reisen führten zu seinem Bruder Wilhelm, der dort Gesandter war. 1817 begleitete ihn Arago, 1818 Valenciennes. Anlaß zur zweiten Italienreise gab der Kongreß von Verona, wohin ihn der preußische König rief. Humboldt begleitete ihn, Friedrich Wilhelm III., nach Venedig, Florenz und Rom und diente ihm als Unterhalter und Erläuterer. Gern hätte ihn der König behalten; aber Humboldt mußte sein Reisewerk in Paris abschließen.

Bewegte Zeitläufte bildeten den politischen Hintergrund zu Humboldts Pariser Aufenthalt. Fast wäre er nach der Schlacht bei Jena als verdächtiger Preuße verhaftet worden, wenn nicht Chaptal ihn in Schutz genommen hätte ${ }^{40}$. Nachdem 
Napoleons Feldzug von 1812 nach Rußland mißlungen war, beteiligte sich Wilhelm von Humboldt am Aufflammen des Aufstandes in Preußen und Österreich. Wilhelm mißbilligte damals den Aufenthalt Alexanders in der feindlichen Hauptstadt $^{41}$. 1814 zogen die Verbündeten in Paris ein; Soldaten sollten ins Musée d'Histoire Naturelle im Jardin des Plantes einquartiert werden. Auf Cuviers Hilferuf eilte Alexander von Humboldt zum preußischen Platzkommandanten und erreichte, daß die Sammlungen verschont wurden. Als der von Elba ausgebrochene Kaiser bei Waterloo endgültig besiegt wurde, zogen die Verbündeten 1815 zum zweitenmal in Paris ein. Wiederum war es Humboldt, der das Museum diesmal mehrfach - vor den Soldaten beschützte ${ }^{42}$.

Mit Ludwig XVIII. war das Königtum zurückgekehrt. 1824 folgte ihm der reaktionäre Karl X. auf den Thron. Chateaubriand, mit dem Humboldt befreundet war, kämpfte für die Pressefreiheit; Arago bekannte freimütig seine liberalen Ansichten ${ }^{43}$. In der Julirevolution von 1830 wurde Karl gestürzt und Louis Philippe als neuer König ausgerufen. Er herrschte bis zur Februarrevolution von 1848. Humboldt hatte in Berlin 1830 über Frankreich vorausgesagt: «Seit vierzig Jahren seh' ich in Paris die Gewalthaber wechseln ... Keine Regierung hat bis jetzt dem Volke Wort gehalten, keine ihre Selbstsucht dem Gemeinwohl untergeordnet. Solange das nicht geschieht, wird keine Macht in Frankreich dauernd bestehen. Die Nation ist noch immer betrogen worden, und sie wird wieder betrogen. ${ }^{44}$

Da Humboldt so lange in Feindesland lebte, mußte es Spannungen zu seiner Heimat geben. Schon 1804 warf man ihm in Preußen vor, er schreibe französisch und lasse sich ins Deutsche übersetzen. «Ein solches Gerücht hat lieblose Quellen », bemerkte er dazu ${ }^{45}$. Der Bruder Wilhelm schrieb aus London, es tue ihm leid, daß Alexander aufgehört habe, deutsch zu sein, und bis in alle Kleinigkeiten pariserisch geworden sei ${ }^{46} .1815$ meldete der "Rheinische Merkur», die Freundschaft der Franzosen habe Humboldt mehr gegolten als die Ehre seines Volkes. Diesmal antwortete Alexander empört. Er drang darauf, daß seine Erwiderung in der Zeitung folge, und fügte bei: «Es muß doch einmal öffentlich gesagt werden, daß der Vorwurf, die Freundschaft eines fremden Volkes der Ehre des seinigen vorzuziehen, mir der härteste von allen erscheint. » ${ }^{47}$ Noch im gleichen Jahr ernannte Minister Hardenberg Humboldt zum preußischen Gesandten in Paris; aber dieser lehnte ab. Er mußte seinem Werk treu bleiben. 1824 wurde er als Vizepräsident der Geographischen Gesellschaft in Paris vorgeschlagen. Daß er auch dieser Ehre auswich, begründete er mit seiner "position d'étranger». ${ }^{48} \mathrm{Er}$ empfand sich also als Deutschen. Er gehörte eben zu den Forschern, die über die Grenzen hinweg dem Fortschritt der Erkenntnis dienten. Er habe sich in zwei 
Sprachen so schön ausgedrückt, daß zwei Völker ihn zu ihren Klassikern rechnen, ist über Humboldt gesagt worden ${ }^{49}$.

1823 weilte er nach langem wieder in Berlin. Der Bruder lebte jetzt dort im väterlichen Schloß Tegel, und nun zum erstenmal fühlte sich auch Alexander angezogen und dachte nach dem Abschied mit Wehmut daran zurück ${ }^{50}$. 1825 hielt er in Paris im Salon der Marquise de Montauban über Monate hin seine Kosmos-Vorlesungen in französischer Sprache ${ }^{51}$. Ein Jahr darauf reiste er nach Berlin, um seine Heimkehr vorzubereiten. Die Ausgabe des Reisewerkes war so weit gediehen, daß er diesen Schritt wagen konnte. Unterwegs besuchte er Goethe. «Er hat an Kenntnissen und lebendigem Wissen nicht seinesgleichen», sagte dieser zu Eckermann ${ }^{52}$. Varnhagen, den Humboldt in Berlin besuchte, wollte nicht an eine Rückkehr glauben. «Für ihn gibt es keinen andern Boden mehr als Paris», vermerkte er in seinem Tagebuch ${ }^{53}$. Aber er täuschte sich. Humboldt kaufte Möbel; von 1827 an lebte er wieder in seiner Heimat Berlin. Hier hielt er die Kosmos-Vorlesungen deutsch; hier leitete er 1828 die Versammlung deutscher Naturforscher und Ärzte; hier konnte der 60 jährige die längst geplante Asienreise verwirklichen und auswerten, hier die Niederschrift des Kosmos, die sein Alter erhellte, bis zum Anfang des fünften Bandes vollenden.

Es war ihm nach dem Tode des Bruders noch ein langes Wirken bis in sein neunzigstes Jahr beschieden; doch kostete er auch viel Bitternis. Jetzt mußte er wirklich als Vorleser und Lexikon des Königs dienen, weil er kein Geld mehr besaß und auf das Gehalt angewiesen war. Varnhagen bedauerte ihn. «Er hat in der Tat wenig Freunde, und nur seine satirische Munterkeit macht ihm das Leben hier noch etwas erträglich. ${ }^{54}$ Die Minister Raumer und Eichhorn waren Reaktionäre; Pietisten und Ultras lebten am Hofe und haßten Humboldt ${ }^{55}$.

In kluger Voraussicht hatte er sich von Anfang an ausbedungen, vier Monate im Jahr in Paris zubringen zu dürfen, und er konnte noch acht letzte Reisen nach Paris unternehmen, vier unter Friedrich Wilhelm III. (1830, 1831, 1835 und 1838), vier unter Friedrich Wilhelm IV. (1841, 1842, 1845 und 1847). 1830 wohnte er im Hôtel d'Angleterre an der Rue Colombier, dann immer im Hôtel de Londres an der Rue des Petits-Augustins 3. In Paris mußte er bei diesen kürzeren oder längeren Aufenthalten immer auch Aufträge des Hofes erfüllen, Louis Philippe besuchen und mit ihm verhandeln. Die Berichte an seinen König waren stilistisch und inhaltlich Meisterstücke ${ }^{56}$.

In seiner Berliner Wohnung «Hinter dem neuen Packhofe» und später an der «Oranienburger Straße» sehnte sich Humboldt oft nach Paris. Varnhagen ist darüber aufschlußreich. Er notiert 1837: «Berlin ist ihm langweilig und drückend, er sehnt sich nach Paris» ${ }^{57}$ und 1843: «Er war in Paris vergnügt und heiter, hier 
hat sich gleich eine trübe Stimmung über ihn gelegt.» ${ }^{58}$ Noch 1847 schreibt Humboldt in Berlin an Josias von Bunsen: "Paris ist mir nicht bloß eine notwendige Erheiterung, da ich hier das belästigte Adreßcomptoir des Landes bin; ich will auch Ideen und Tatsachen zu dem dritten und letzten Bande des 'Kosmos' einsammeln. ${ }^{59}$ Laut seiner eigenen Aussage erhielt Humboldt im Jahr 3000 Briefe, von denen er etwa 2000 beantwortete ${ }^{60}$.

Paris blieb für Humboldt die Pflanzstätte der modernen Naturwissenschaften. Dort hatte er den Grund zu der Breite seines Wissens gelegt. Er nahm innigen Anteil an den Schicksalen des Nachbarlandes, und als 1852 nach einem blutigen Staatsstreich mit Verbannungen Louis Napoleon sich zum Kaiser aufschwang, nannte er ihn einen gewissenlosen Abenteurer und sah einen Krieg mit Deutschland voraus ${ }^{61}$. Diese Prophezeiung hat sich 1870 erfüllt. Flüchtlinge, so den Bildhauer David d'Angers, empfing Humboldt in seiner Wohnung. «Glänzende Programme von Versprechungen und Reformen sollen das Volk über den Verlust seiner Freiheit täuschen », sagte er $^{62}$. Als man den Greis eines Tages fragte, ob es wahr sei, daß er nach Südfrankreich zur Erholung fahre, brach er aus: «Ach, das ist wieder eine jener von Zeit zu Zeit über mich in Umlauf gesetzten Mythen. Was soll ich im südlichen Frankreich tun? Reiste ich überhaupt, so würde ich nach Paris zu Arago gehn, der mich mehr interessiert als alle klimatischen Einflüsse auf meine Gesundheit. ${ }^{63}$

Humboldt war einer der besten Menschenkenner seiner Zeit. Dies ermöglichte ihm auch die Auffindung besonderer Begabungen. Es ist erstaunlich, wie viele Genies er entdeckt und gefördert hat. Die führenden Chemiker der Jahrhundertmitte, Jean-Baptiste Dumas in Frankreich und Justus Liebig in Deutschland, waren beide Entdeckungen Humboldts ${ }^{64}$. 1822 saß der Apothekerlehrling Dumas in seiner Kammer in Genf, als unversehens Humboldt, unterwegs nach Verona, bei ihm eintrat. Er hatte seine Abhandlung über das Blut gelesen und erzählte ihm nun von den großen Pariser Chemikern, so daß Dumas zu ihnen aufbrach ${ }^{65}$. Liebig hielt 1824 als unbekannter Student in Paris einen Vortrag über Silber- und Quecksilberverbindungen. Als er seine Präparate zusammenpackte, knüpfte ein älterer Herr mit ihm ein Gespräch an und lud ihn für den nächsten Tag ein. «Diese Unterhaltung ist der Grundstein meiner Zukunft gewesen», schreibt Liebig ${ }^{66}$. Zwar gelang die zweite Begegnung nicht sogleich, da Humboldt vergessen hatte, sich vorzustellen. Aber bald darauf stellte Humboldt den jungen Forscher Gay-Lussac und Thenard vor. «Von diesem Tage an waren mir alle Türen, alle Institute und Laboratorien geöffnet. ${ }^{67}$

Carl Vogt hat von den «Dachstubenstunden» Humboldts erzählt, in denen dieser in Paris bewußt nach Begabungen suchte ${ }^{68}$. So stieß er auch auf den 
Schweizer Zoologen Louis Agassiz. Dieser berichtet: «Nach einer herzlichen Begrüßung ging er sogleich auf das los, was damals meine Bibliothek war.» Humboldt lud ihn zum Abendessen ein. «Und für drei Stunden, die mir wie ein Traum vorüberrauschten, hatte ich ihn ganz für mich allein. Wie fragte er mich aus, und wieviel lernte ich in der kurzen Zeit! Wie ich arbeiten, was ich tun, was ich lassen müsse, wie leben, wie meine Zeit einteilen, welche Wege des Studiums einschlagen. ${ }^{69}$

Humboldt hat Liebig die Professur in Gießen, Agassiz jene in Neuenburg verschafft. Ein junger Freund hat über Humboldt geschrieben: «Mit ihm zu verkehren war im wahren Sinne ein Wandeln auf den Höhen der Menschheit. ${ }^{70}$

Der große Naturforscher verbrachte den vierten Teil seines Lebens in Paris. Dort erschien sein Hauptwerk, die Ausbeute der amerikanischen Reise. Dort nur standen ihm die Forscher zur Verfügung, die ihm die mitgebrachten Meßwerte und Sammlungen bearbeiten halfen. In der Kriegszeit suchte er Versöhnung und nicht Vergeltung und rettete das Museum vor dem Zugriff seiner Landsleute. Erst der 58jährige kehrte nach Berlin zurück. Aber noch achtmal besuchte er Paris - «die Stadt, die ihm die zweite und oft die wahre Heimat gewesen war» (Hanno Beck) ${ }^{71}$. Das Schönste, was Frankreich ihm bescherte, war der Umgang mit Gelehrten, zu denen er emporsah, wie Lagrange und Berthollet, mit Gefährten, die seine Forschung teilten, wie Bonpland und Gay-Lussac, und mit Freunden, die seinen Weg begleiteten, wie Arago und Valenciennes. In Humboldt haben sich Deutschland und Frankreich beispielgebend verbunden.

\section{Lebensdaten der erwähnten französischen Forscher}

Arago, François, Astronom und Physiker, 1786-1853

Berthollet, Claude-Louis, Chemiker, 1748-1822

Biot, Jean-Baptiste, Physiker, 1774-1862

Bonpland, Aimé, Botaniker, 1773-1858

Borda, Jean-Charles, Physiker, 1733-1799

Boussingault, Jean-Baptiste, Chemiker und Agronom, 1802-1887

Brongniart, Alexandre, Geologe, 1770-1847

Champollion-Figeac, Jacques-Joseph, Archäologe, 1778-1867

Champollion, Jean-François, Hieroglyphenforscher, 1790-1832

Chaptal, Jean-Antoine, Chemiker, 1756-1832

Cuvier, Georges, Paläontologe, 1769-1832

Delambre, Joseph, Astronom, 1749-1822

Desfontaines, René Louiche, Botaniker, 1750-1833

Dujardin, Félix, Zoologe, 1801-1860 
Dumas, Jean-Baptiste, Chemiker, 1800-1884

Fourcroy, Antoine-François de, Chemiker, 1755-1809

Fourier, Joseph, Mathematiker, 1768-1830

Gay-Lussac, Louis-Joseph, Chemiker und Physiker, 1778-1850

Geoffroy Saint-Hilaire, Etienne, Zoologe, 1772-1844

Jomard, Edme-François, Geograph, 1777-1862

Jussieu, Antoine-Laurent de, Botaniker, 1748-1836

Lagrange, Joseph-Louis, Mathematiker, 1736-1813

Lalande, Jérôme de, Astronom, 1732-1807

Lamarck, Jean-Baptiste, Botaniker und Zoologe, 1744-1829

Laplace, Pierre-Simon, Mathematiker, 1749-1827

Latreille, Pierre-André, Zoologe, 1762-1833

Letronne, Jean-Antoine, Archäologe, 1787-1848

Poisson, Siméon-Denis, Mathematiker, 1781-1840

Richard, Louis-Claude-Marie, Botaniker, 1754-1821

Sacy, Antoine-Isaac, baron Silvestre de, Orientalist, 1758-1838

Thenard, Louis-Jacques, Chemiker, 1777-1857

Valenciennes, Achille, Zoologe, 1794-1865

Vauquelin, Louis-Nicolas, Chemiker, 1763-1829

\section{Quellenhinweise}

Hauptquellen und ihre Sigel:

- Beck, Hanno, Gespräche Alexander von Humboldts, Berlin 1959 (zitiert: Gespräche).

- Beck, Hanno, Alexander von Humboldt, 2 Bände, Wiesbaden 1959 und 1961 (zitiert: Beck I und II).

- Biermann, Kurt-R., Jahn, Ilse, und Lange, Fritz G., Alexander von Humboldt. Chronologische Übersicht über wichtige Daten seines Lebens, Berlin 1968 (zitiert: Chronologie).

- Bruhns, Karl (Hrsg.), Alexander von Humboldt. Eine wissenschaftliche Biographie. 3 Bände, Leipzig 1872 (zitiert: Bruhns).

- Scurla, Herbert, Alexander von Humboldt, 3.Auflage, Berlin 1960 (zitiert: Scurla).

- Terra, Helmut de, Alexander von Humboldt und seine Zeit, Wiesbaden 1956 (zitiert: de Terra).

1 Alexander von Humboldt, Kosmos, 4 Bände, Stuttgart/Augsburg 1845-1858, Band 2, S. $67 \mathrm{f}$.

${ }^{2}$ Chronologie, S. 3.

${ }^{3}$ Beck I, S. 27.

${ }^{4}$ Gespräche, S. 15.

${ }^{5}$ Gespräche, S. 319. - Joseph E. Hofmann, Alexander von Humboldt in seiner Stellung zur reinen Mathematik und ihrer Geschichte. In: Alexander von 
Humboldt, Gedenkschrift zur 100. Wiederkehr seines Todestages, hrsg. von der Alexander von Humboldt-Kommission der Deutschen Akademie der Wissenschaften zu Berlin, Berlin 1959, S. 247 f.

${ }^{6}$ Beck I, S. 115.

7 Beck I, S. 117.

${ }^{8}$ Beck II, S. 11.

${ }^{9}$ Scurla, S. 212.

${ }^{10}$ Bruhns I, S. 472-480, II, S. 79; Scurla, S. 253 f.

11 Bruhns II, S. 5.

12 Vgl. in: Chronologie. Dort sind auch die Wohnadressen vermerkt.

13 Zum Reisewerk: Bruhns II, S. 17-21; de Terra, S. 159-161; Scurla, S. 214-224; Beck II, S. 68-76.

14 Kurt-R.Biermann, Lagrange im Urteil und in der Erinnerung A. von Humboldts. Monatsberichte der Deutschen Akademie der Wissenschaften zu Berlin 5 (1963) 445-450. Zitat von 1846 auf S. 447.

${ }^{15}$ Felix Klein, Vorlesungen über die Entwicklung der Mathematik im 19. Jahrhundert, 2 Bände, Berlin 1926 und 1927 (Neudruck: New York 1950), Band 1, S. 17.

${ }^{16}$ Bruhns II, S. 59 f.; Chronologie, S. 59 (Champollions Beisetzung).

17 Bruhns II, S. $34 \mathrm{f}$.

${ }_{18}$ Franz Aragos Werke (16 Bände, Leipzig 1854-1860), Band 3, S.16.

${ }^{19}$ Humboldt und Gay-Lussac, Das Volumgesetz gasförmiger Verbindungen. Ostwalds Klassiker Nr. 42, Leipzig 1921, S.17.

${ }^{20}$ Zum Folgenden vgl. Franz Aragos Werke, Band 1, S. 31-67, und Band 15, Plan der vermessenen Dreiecke bei S. 48.

${ }^{21}$ Beck II, S. 27; de Terra, S. 165.

${ }^{22}$ Brief an Christian Heinrich Schumacher, zitiert nach Bruhns II, S. 32.

${ }^{23}$ Brief vom 24. Januar 1829 an Geoffroy Saint-Hilaire, zitiert nach Bruhns II, S. 32 .

${ }^{24}$ Brief an den Architekten Jacques-Ignace Hittorff, zitiert nach Bruhns II, S.33.

25 Gespräche, S. 200.

${ }^{26}$ Kurt-R.Biermann, Die Beziehungen Alexander von Humboldts zu französischen Mathematikern. Monatsberichte der Deutschen Akademie der Wissenschaften zu Berlin 11 (1969) 458-463. Darin S. 461.

27 Gespräche, S. 168, 172 und S. 385.

28 de Terra, S. 169; Bruhns II, S. 61.

${ }^{29}$ Gespräche, S. XX.

30 Wilhelm von Humboldt an seine Frau, 30. November 1815 (Gespräche, S. 47).

${ }^{31}$ Chamisso an Eduard Hitzig, 16. Februar 1810 (Gespräche, S. 37).

32 Gespräche, S. 70 f.

${ }^{33}$ Verzeichnet in: Chronologie (von 1798 bis 1832).

${ }^{34}$ Gespräche, S. 68.

35 Bruhns II, S. $28 \mathrm{f}$.

${ }^{36}$ Erzählt von Carl Vogt (Gespräche, S. 203-206).

37 Scurla, S. 227.

38 Ebendort. 
39 Alexander von Humboldt, Ansichten der Natur, 2 Bände, Stuttgart/Tübingen 1808,3 . Auflage 1849, S. IX f.

40 Beck II, S. 27.

41 Beck II, S. 35.

42 Beck II, S. 36 und $38 \mathrm{f}$.

43 de Terra, S. 190.

44 Gespräche, S. 122.

45 Scurla, S. 219.

16 Wilhelm von Humboldt an seine Frau, 3.Dezember 1817 (Gespräche, S. 52).

${ }^{47}$ Chronologie, S. 41 ; Beck II, S. $40 \mathrm{f}$.

${ }^{48}$ Brief an Konrad Malte-Brun, 27. März 1824 (Bruhns II, S. 55).

${ }^{49}$ Gespräche, S. 369.

50 Beck II, S. 60.

${ }^{51}$ Chronologie, S. 47.

52 Johann Peter Eckermann, Gespräche mit Goethe, 11. Dezember 1826.

53 Varnhagen, 18. November 1826 (Gespräche, S. 84).

54 Scurla, S. 325.

55 Varnhagen, 26. Dezember 1845 (Gespräche, S. 215).

56 Beck II, S. 161.

57 Varnhagen, 2.April 1837 (Gespräche, S. 161).

58 Varnhagen, 18. März 1843 (Gespräche, S. 191).

${ }^{59}$ Brief vom 3. Oktober 1847 (Scurla, S. 364).

60 Angabe von 1856 (Gespräche, S. 374).

${ }^{61}$ Gespräche, S. 325, 336, 412.

${ }^{62} \mathrm{Zu}$. Friedrich Althaus, 5. August 1852 (Gespräche, S. 341).

${ }^{63} \mathrm{Zu}$ Friedrich Althaus, 11. Dezember 1851 (Gespräche, S. 312).

${ }^{61} \mathrm{Vgl}$. August Wilhelm von Hofmann in: Gespräche, S. 75 Mitte.

${ }^{65}$ Günther Bugge (Hrsg.), Das Buch der großen Chemiker, 2 Bände, Berlin 1929 und 1930, Band 2, S. 58 f.; Gespräche, S. 73-75.

${ }^{66}$ Bruhns II, S. 68; auch in Wilhelm Ostwald, Große Männer, 2. Auflage, Leipzig 1910, S. 162.

67 Ebendort.

${ }^{68}$ Bruhns II, S. 62; Gespräche, S. 206.

${ }^{69}$ Bruhns II, S. $211 \mathrm{f}$.

${ }^{70}$ Friedrich Althaus, 5. August 1852 (Gespräche, S. 340).

${ }^{71}$ Beck II, S. 185.

Herr Prof. Hanno Beck regte das Thema an, und Herr Prof. Ackerknecht übersetzte das Summary; beiden danke ich.

In Zitaten wurde die heutige Rechtschreibung verwendet. 


\section{Summary}

Alexander von Humboldt saw Paris first in 1790, together with George Forster. He remained faithful to the ideals of liberty of those days. The second time he was in Paris in 1798, when preparing an expedition. There he found Aimé Bonpland, his companion during his American trip (1799-1804). After his return he found there the collaborators of this 30 volume report. While Napoleon conquered Prussia Humboldt lived in his fatherland, but then he returned to Paris. The contacts with Lagrange and other scientists, the friendship of Gay-Lussac and Arago helped him. He regularly spoke in the Académie des Sciences. He participated in the meetings of Berthollet's Société d'Arcueil. Humboldt's brother Wilhelm was afraid he would loose him to Paris. But he returned to Berlin in 1827. He visited Paris still 8 more times, the last time at the age of 78 . It remained the city of his yearning, which had given him the most productive years of his life.

Dr. phil. Heinz Balmer

Medizinhistorisches Institut

Rämistraße 71

8006 Zürich 